\title{
Sistemas de información geográfico y la preservación del patrimonio natural y cultural: la exploración de La Mosquitia hondureña
}

Juan Carlos Fernandez-Diaz, Steve Elkins, Alicia M. González, William Carter, Ramesh Shrestha, Michael Sartori, Abhinav Singhania, Craig, Glenie, Bill Benenson, Garry Spire

\section{Resumen:}

En Mayo del 2012, después de más de un año de investigación y planeación, un grupo dirigido por el cinematógrafo Steve Elkins con el respaldo de Bill Benenson, y Garry Spire, realizó el primer mapeo exploratorio de la Mosquitia Hondureña utilizando tecnología LiDAR abordo un avión bimotor Cessna 337. La tecnología LiDAR, que es un acrónimo en Inglés para Light Detection and Ranging, es también conocida como Radar Óptico o Radar Laser y utiliza pulsos laser para generar mapas tridimensionales de la superficie terrestre. En general, equipos LiDAR actuales permiten detectar múltiples retornos para cada pulso emitido a medida que estos se propagan a través de aberturas en las coronas de los árboles y demás vegetación hasta el terreno debajo de ella. A través de filtros morfológicos es posible clasificar los retornos como provenientes de vegetación o del suelo, lo que permite separar los retornos del suelo revelando lo que el bosque oculta. La Mosquita provee las condiciones más difíciles para probar las capacidades de la tecnología LiDAR.

La planeación, recolección, procesamiento y análisis preliminar de los datos LIDAR fue efectuada por técnicos del centro de investigación de ciencias e ingeniería de sistemas de Geopercepción de la Universidad de Houston y el Centro Nacional para el Mapeo Laser Aéreo de la Fundación Nacional para la Ciencia. El mapeo se enfocó en varias aéreas dentro de una extensión de jungla de aproximadamente $1000 \mathrm{~km}^{2}$ en el departamento de Gracias a Dios. Los planes de vuelo y el equipo fueron configurados para lograr la máxima penetración a través de la vegetación. Emitiendo más de 20 pulsos $/ \mathrm{m}^{2}$ y obteniendo aproximadamente 31 retornos $/ \mathrm{m}^{2}$ de 
los cuales solo 1 o 2 corresponden al suelo. Los modelos digitales de elevación de terreno revelan debajo de la espesa vegetación una exuberante topografía y una muy compleja hidrografía. Mas interesante, muestran una extensa modificación antropogénica del terreno, en lo que parece ser restos arqueológicos.

Palabras clave: LiDAR/ Radar Óptico/ Sistemas de Información Geográfico/ Arqueología/ Mosquitia Hondureña.

\section{Abstract}

In May 2012, after more than a year of research and planning, a group led by cinematographer Steve Elkins and backed by Bill Benenson and Garry Spire, conducted the first ever airborne LiDAR exploratory survey of the Honduran Mosquitia region utilizing a Cessna 337 twin engine airplane. LiDAR technology, which is an acronym for Light Detection and Ranging, is also known as Optical Radar or laser Radar and it employs laser pulses to generate three-dimensional maps of the of the Earth's surface. Current LiDAR units can generally record multiple returns per laser shot, and as the pulses propagate through partial openings in the forest canopy, they produce returns from the vegetation, the underlying terrain, or both. Through the use of morphological filters it is possible to classify returns as coming from the vegetation or ground, and the ground returns reveal what is hidden under the forest canopy. La Mosquitia jungle provides the toughest conditions on which to test airborne LiDAR capabilities.

The LiDAR data collection, processing and preliminary analysis were performed by researchers from the University of Houston (UH) Geosensing Engineering and Science Research Center and the National Science Foundation National Center for Airborne Laser Mapping (NCALM). The survey was focused in several areas within a jungle area of roughly $1000 \mathrm{~km}^{2}$ in the department of Gracias a Dios. The flight plans and equipment were configured to maximize canopy penetration. Firing more than 20 laser pulses $/ \mathrm{m}^{2}$, which yielded approximately 31 returns $/ \mathrm{m}^{2}$, only 1 or 2 of which were found to be ground returns. Under the forest canopy, the digital elevation models reveal an exuberant topography and a very complex hydrology. Perhaps even more interesting, they reveal extended anthropogenic terrain modifications in what appear to be archeological remains.

Key words: LIDAR/ Optical Radar/ Geographic Information Systems/ Archeology/ Honduran Mosquitia. 
Juan Carlos Fernandez-Diaz1 (jfernan4@central.uh.edu), University of Houston / NSF National Center for Airborne Laser Mapping; Steve Elkins, UTL Scientific / UTL Productions; Alicia M. González, William Carter, University of Houston / NSF National Center for Airborne Laser Mapping; Ramesh Shrestha, University of Houston / NSF National Center for Airborne Laser Mapping; Michael Sartori, University of Houston / NSF National Center for Airborne Laser Mapping; Abhinav Singhania, University of Houston / NSF National Center for Airborne Laser Mapping; Craig, Glenie, University of Houston / NSF National Center for Airborne Laser Mapping; Bill Benenson, Garry Spire. 


\section{INTRODUCCIÓN}

Frecuentemente llamada los pulmones de Centro América o la Pequeña Amazonia, la región de la Mosquitia Hondureña es una de las pocas junglas tropicales vírgenes en existencia hoy en día. Caracterizada por una exuberante y abundante flora y fauna, con un terreno muy variado compuesto por altas montañas, sabana de pinos dispersos, humedales, manglares y una extensa red de ríos, riachuelos, lagunas y pantanos. Durante los últimos 500 años bajo el dominio Español y Británico, múltiples y continuos intentos de exploración y explotación han brindado muy pocos resultados y la mayor parte de la región se mantiene desconocida, inexplorada y sin mapas precisos y confiables, esto debido a la naturaleza agreste del terreno y la vegetación. Desde hace mucho tiempo la Mosquitia ha sido valorada por sus recursos naturales y desde 1980 forma parte de la Red Mundial de Biosferas de la UNESCO. Esfuerzos metódicos y sistemáticos para el mapeo y levantamiento de inventario del patrimonio natural son continuos. Sin embargo a pesar de su estatus protegido tanto a nivel nacional como internacional, la Mosquitia se encuentra bajo constante asecho por parte de depredadores del bosque y la naturaleza, cazadores de tesoros y más recientemente narcotraficantes.

En cuanto al patrimonio cultural de la región, lo que se conoce es minúsculo en comparación con el tamaño de la región y el legado de los habitantes indígenas de la región no ha sido completamente valorado. Los intentos de colonización y/o asentamiento en sus tierras tradicionales son temas recurrentes en las historias orales y escritas desde tiempos ancestrales. La región que es compartida entre Honduras y Nicaragua ha sido el hogar para múltiples grupos que han sido ligados con algunos de los idiomas y lenguajes más antiguos de Norte y Sur América. Algunas estimaciones no verificadas, ubican estos grupos étnicos en la zona en una época aun tan temprana como el Periodo Arcaico de entre 4000 a 2000 años Antes de la Era de Cristo. Aun y cuando, los habitantes de la Mosquitia en el Siglo 16 nunca fueron conquistados por los españoles, su influencia y la influencia de las subsecuentes incursiones e interacciones resultaron en nuevos y complejos grupos étnicos y culturales como los Misquitos. Grupos que son el resultado del comercio a lo largo de la cuenca del mar Caribe, y la mezcla y mestizaje de los pobladores autóctonos, africanos y europeos. Estas intrusiones por los foráneos, que quizás ocurrieron durante muchos siglos, dispersaron o quizás eliminaron a los pobladores autóctonos de la región. Grupos de los que se sabe han habitado y en algunos casos continúan habitando la región incluyen los Tawaka, Sumo, Pech (anteriormente 
conocidos como Paya), los Misquitos, los Garífunas, los Tolupanes; y los Rama y Ulwa (mas que todo en Nicaragua). El patrimonio cultural de estos pueblos, el cual es tanto tangible como intangible es igual de variado y misterioso como la región misma. El patrimonio intangible, incluye historias de los vínculos ancestrales con el terreno, la jungla, sus lugares sagrados, historias de grandes ciudades fortificadas, historias que muchos clasifican como mitos o leyendas. Sin embargo, en raras ocasiones elementos del patrimonio tangible son descubiertas y sirven para enriquecer los mitos y leyendas. Pero a estas alturas, la mayor parte del patrimonio cultural permanece oculto, desconocido y los pocos fragmentos de los que se conocen proveen más preguntas que las respuestas que proporcionan.

La investigación de campo de manera sistemática en la región de la Mosquitia es extremadamente difícil. Arqueólogos y antropólogos asociados con el Instituto Hondureño de Antropología e Historia han hecho esfuerzos de exploración e investigación desde la fundación del instituto hace ya más de 60 años con resultados muy pequeños comparados con otras regiones menos precarias de Honduras. A través de la tenacidad y persistencia de un puñado de individuos, varios sitios arqueológicos han sido descubiertos, documentados y registrados como patrimonio cultural de la región. Sitios como Las Crucitas de Aner, El Higuerito, El Lancetillal, Los Metates del Camotal, y los petroglifos de Walpulbansirpe. Estos pocos sitios, son solo una pequeña fracción de de lo que puede ser una red extensa de comunidades en la región, creando más preguntas que respuestas. Ahora, la tecnología de Mapeo LIDAR se presta como una herramienta para facilitar la identificación del extenso patrimonio natural y cultural de la región y para brindar algunas respuestas a las múltiples interrogantes y leyendas de la región.

\section{BREVE RESEÑA DE LA EXPLORACIÓN DE LA MOSQUITIA}

Una de las primeras expediciones de exploración de la Mosquitia Hondureña de las que hay registro histórico fue descrita en una carta al Rey de España por el Obispo Cristóbal de Pedraza. Dicha carta relata un arduo viaje hacia el borde de la jungla de la Mosquitia. Más recientemente en 1915, el profesor y cartógrafo Jesús Aguilar Paz inició su recorrido alrededor de Honduras para producir el primer mapa moderno y completo del país. Dicho mapa fue publicado en 1933 y en su recorrido el Dr. Aguilar Paz visito las distintas comunidades de la Mosquita. (Mugnier, 1999) La primera expedición arqueológica de la región fue realizada en 1933 por el arqueólogo William Duncan Strong del instituto Smithsonian. En su diario de campo, Strong 
documenta la existencia de montículos arqueológicos en la zona, entre muchos los montículos de Wankibila o Guanquivila en las orillas del Rio Patuca y los montículos de Floresta a las orillas del río Conquirre. (Smithsonian Institution, SF) Más cercano a nuestro tiempo, arqueólogos y antropólogos como George Hasemann (ya difunto), Gloria Lara Pinto y Chris Begley han identificado y documentado cientos de pequeños pero muy interesantes sitios arqueológicos en la región. (Lara-Pinto, 2006) (Begley, 2002 y 2007) Sin embargo, hasta la fecha ningún complejo arqueológico de gran tamaño descrito en las leyendas ha sido identificado a pesar de los múltiples esfuerzos por arqueólogos y cazadores de tesoros. Tecnologías de percepción remota desde plataformas satelitales incluyendo imágenes ópticas y Radar de Apertura Sintética (Yakam-Simen et al., 1999) han sido empleadas por algunos para facilitar la exploración de la región sin producir notables resultados.

\section{LA TECNOLOGÍA LIDAR}

LiDAR que es un acrónimo en Inglés para Light Detection And Ranging 0 detection y medición de distancia por luz, es también conocido como radar óptico, al utilizar pulsos de luz visible en vez de pulsos de radiofrecuencia para detectar objetos y medir la distancia entre el equipo y dicho objeto. El LiDAR es una técnica de percepción remota que utiliza radiación electromagnética en la longitud de ondas visibles para detectar objetos, determinar la distancia al objeto y también permite deducir propiedades físicas del objeto con base en las interacciones entre la radiación y el objeto. Hay muchas formas y aplicaciones del LiDAR, ejemplos incluyen el mapeo de la superficie terrestre y subacuática, la estructura del bosque, la detección de componentes y contaminantes atmosféricos, la determinación de la temperatura del aire, la velocidad y dirección de viento a diferentes niveles de la atmosfera, visión computarizada y detección de obstáculos para robots y vehículos autónomos, etc. (Fernandez-Diaz et al., 2013). En este trabajo se trata exclusivamente con LIDAR de mapeo aéreo, también conocido como Airborne LiDAR Swath Mapping o ALSM. Los primeros sistemas de LiDAR de mapeo aéreo, desarrollados a mediados de la década de 1960, funcionaron en modo altímetro de perfilamiento, es decir que medían y registraban la altitud relativa del avión respecto al terreno justo debajo del avión en forma de perfil y utilizaban un barómetro para registrar la altitud respecto al nivel medio del mar. Aplicaciones de estos sistemas incluían la medición del grosor de las capas de hielo en el océano ártico y la detección de submarinos en aguas poco profundas. (Hickman y Hogg, 1969) Este interés militar empujó el desarrollo de sistemas para aplicación batimétricas durante más de una 
década. En 1977 se inicio a trabajar en el primer sistema LiDAR que empleaba un espejo de barrido, el que permitía dirigir el haz laser en un pequeño ángulo hacia los costados del avión, perpendicularmente a la dirección de vuelo. Esto permitió al sistema LiDAR conocido como Airborne Oceanographic LiDAR o AOL registrar la elevación de una franja de superficie en vez de un simple perfil. (Gunter et al. 1978) Los primeros resultados en los que el AOL fue utilizado para registrar la elevación de la superficie terrestre y la vegetación fueron reportados en 1984. Desde entonces, hasta esta fecha múltiples avances en tecnologías claves como sistema de navegación por satélite, unidades de medición inercial (IMUs), láseres pulsados, foto detectores y sistemas de cómputo sofisticados han convertido el LIDAR de mapeo en el método más preciso y de mayor resolución para mapeo tridimensional de la topografía terrestre.

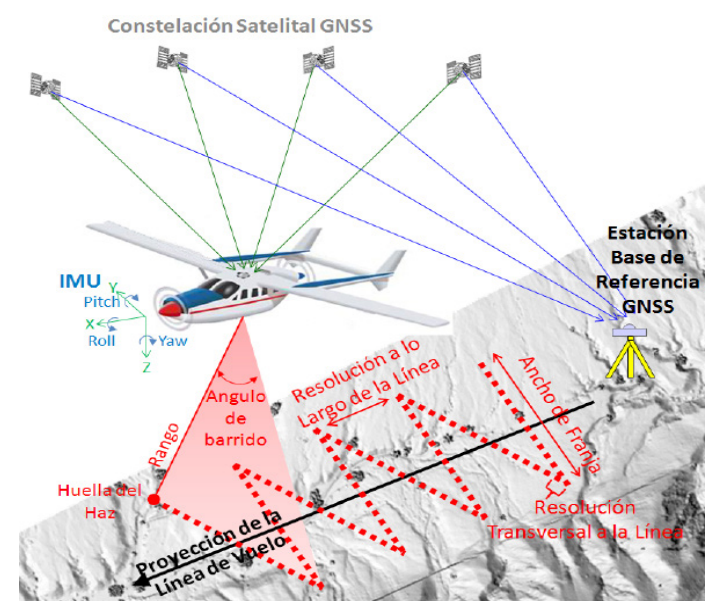

Figura 1. LiDAR de Mapeo Aéreo.

Los principios básicos del LIDAR de mapeo aéreo se ilustran en la Figura 1. La parte más importante del sistema, es una fuente laser pulsada, que emite pulsos laser de corta duración (FWHM 10 nanosegundos) y que se repiten miles de veces por segundo en lo que se llama frecuencia de repetición de pulso (PRF). Estos pulsos laser salen del avión a través de un puerto de mapeo en el fuselaje del avión y son distribuidos en 2 dimensiones sobre el área de interés. La primera dimensión es a lo largo de la dirección de vuelo del avión y se logra con el movimiento propio del avión. 
La segunda dimensión se logra a través de un mecanismo de barrido, generalmente un espejo rotante u oscilante, que orienta los pulsos laser hacia los costados del avión perpendicularmente a la dirección de vuelo. La combinación del movimiento propio del avión y el barrido por el espejo distribuyen los pulsos laser sobre el terreno en un patrón con forma de diente de sierra. La selección del ángulo de barrido del espejo y la altitud de vuelo sobre el terreno determinan el ancho de la franja de mapeo. La frecuencia de barrido, el PRF determina la separación entre pulsos laser sobre el terreno en la dirección perpendicular a la línea de vuelo, o resolución transversal a la línea. La velocidad sobre el terreno del avión y la frecuencia de barrido determinan la separación de los pulsos laser sobre el terreno en la dirección del vuelo, o resolución a lo largo de la línea.

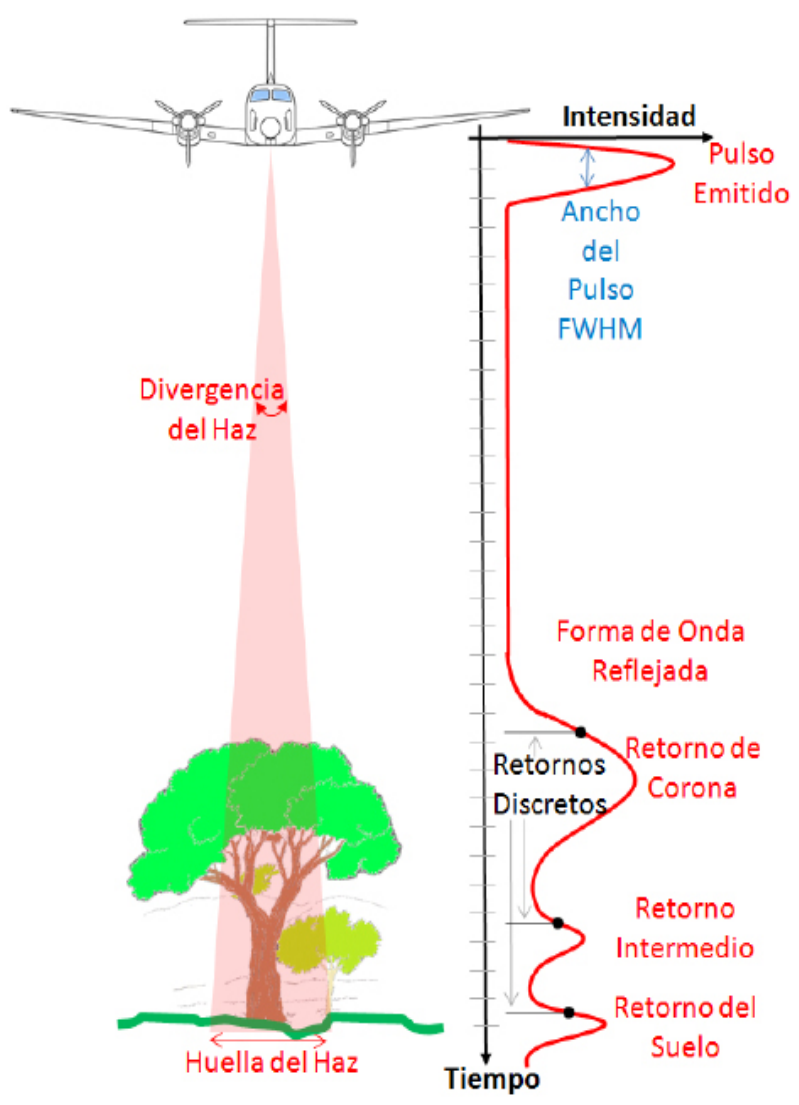

Figura 2. Propagación de un Pulso Laser. 
La Figura 2 ilustra que la energía laser de un único pulso se propaga a través de la atmósfera en una forma cónica, similar al patrón de radiación de un foco de mano altamente directivo. El ángulo en el que se dispersa el haz laser depende de la divergencia óptica del laser y en conjunción con el altura de vuelo determinan que tan grande es la "huella del haz" en el suelo. La mayor parte de los sistemas de mapeo aéreo tienen divergencia que producen huellas de haz con diámetros de entre $20 \mathrm{~cm}$ a $1 \mathrm{~m}$, por ello se categorizan como sistemas de huella pequeña, en contraste a sistemas satelitales que tienen huellas de $30 \mathrm{~m}$ de diámetro. La Figura 2 también ilustra un gráfico de la intensidad de la luz laser emitida y recibida en función del tiempo de propagación, lo que se conoce como forma de onda.

La forma de onda de un pulso laser al salir del sensor LiDAR es un perfil Gaussiano, y a medida que el pulso interactúa con la vegetación o el terreno parte de la energía es retornada hacia el sensor, modificando la forma de onda dependiendo de las propiedades geométricas y espectrales del objeto que interactúa con el pulso. Los fotones que son reflejados de regreso al sensor, son detectados por un foto detector, generalmente un fotodiodo de avalancha (APD) o un tubo fotomultiplicador (PMT). La señal producida por el foto detector puede ser digitada y almacenada para posterior análisis o puede ser analizada en tiempo real por un circuito analógico que produce marcas de tiempo muy precisas para eventos muy particulares. Del análisis de las formas de onda o de las marcas de tiempo se puede determinar el tiempo de vuelo (ida y regreso) entre el pulso emitido y los distintos elementos que reflejaron parte de la energía de regreso al sensor. Dividiendo este tiempo de vuelo entre 2 y multiplicándolo por la velocidad de la luz para el medio adecuado, se determina la distancia entre el sensor y el objeto que produjo la reflexión de la energía (retorno), o el rango entre el sensor y el objeto. Conociendo la posición y la orientación del avión, es posible producir coordenadas 3-dimensionales para cada uno de los objetos que produjeron un retorno.

Como se ilustra en la Figura 1, hay 2 dispositivos que forman parte de un sistema de mapeo LiDAR que sirven para determinar la posición y la orientación del sistema para georeferenciar los retornos. El primer componente es un receptor de calidad geodésica del sistema satelital de navegación global o GNSS. Adicionalmente al receptor GNSS es necesario contar con por lo menos un receptor en una ubicación fija o una estación base de referencia. Dichas estaciones de referencia se deben de localizar a una distancia máxima de $100 \mathrm{~km}$ de la región de mapeo. Todos los receptores del sistema tienen que funcionar simultáneamente y almace- 
nan la información que reciben de los satélites en memoria para efectuar un procesamiento post-vuelo. Las estaciones base almacenan datos por un largo periodo (> 4 horas), lo que permite determinar la posición de la estación con una precisión mejor que un centímetro tanto en la orientación horizontal y vertical. Una vez que la posición de las estaciones base han sido determinadas, se pueden procesar las observaciones GNSS del avión y de las estaciones de forma vectorial diferencial para determinar la trayectoria del avión (latitud, longitud y elevación o XYZ) con referencia a las estaciones bases. El segundo componente es la unidad de medición inercial (IMU), la que está compuesta de 3 acelerómetros y 3 giroscopios que se ubican en una triada ortogonal. La IMU registra las aceleraciones lineares y angulares del avión alrededor de 3 ejes longitudinal (roll), lateral (pitch) y vertical (yaw). La trayectoria diferencia GNSS y la información registrada por la IMU se combinan en un filtro de estimación óptima (Kalman Filter) para producir la mejor estimación de trayectoria completa (posición y orientación) en lo que se conoce como SBET (Smoothed Best Estimate of Trajectory).

La trayectoria completa posteriormente se combina con la información de tiempos, distancias e intensidades de cada retorno obtenidas con el sistema LiDAR y se produce el primer producto de datos, la nube de puntos (PC). La nube de puntos es una colección de coordenadas, estampas de tiempo, intensidad y otra información del sistema (tipo de retorno, clase y ángulo de barrido) para cada retorno detectado por el sistema. La nube de puntos es información geo-espacial de naturaleza irregular, ya que la separación entre los retornos no es uniforme, más bien siendo un proceso completamente aleatorio. Como se ilustra en la Figura 2, cada pulso laser emitido por el sistema puede producir múltiples retornos discretos. La combinación de longitud de onda, divergencia de haz, y la capacidad de barrer el haz laser permite que la energía laser se propague a través de aberturas en la corona de los árboles y así produce múltiples retornos a diferentes niveles de la vegetación y del suelo. Cada uno de estos retornos representa un elemento único en la nube de puntos.

El siguiente paso en el procesamiento de datos LiDAR consiste en clasificar cada retorno de la nube de punto como producido por vegetación, por el suelo o por algún tipo de edificación. La clasificación de los retornos como producidos por el terreno u otro objeto se realiza con un filtro morfológico que inicia identificando los retornos con la elevación más baja en cada celda de una cuadricula de búsqueda. El tamaño de las celdas en la cuadrícula es seleccionado de manera que coincida 
con el máximo tamaño de edificaciones a encontrar en el aérea. De esta manera, es muy probable que los retornos con la elevación más baja seleccionados para cada cuadrícula sean retornos provenientes del suelo. A partir de aquí se inicia un proceso iterativo de construir facetas trianguladas entre los puntos clasificados como suelo y evaluar la elevación de cada retorno contra la respectiva elevación de la superficie triangulada. Si la elevación del retorno es superior a la elevación de la superficie, es muy probable que no corresponda al suelo, en cambio si su elevación es inferior a la superficie es muy probable que si corresponda al suelo. Con cada iteración el número de puntos clasificados como suelo aumentan, densificando el número de superficies triangulares y reduciendo sus áreas, hasta que llega un punto que no se pueden agregar más puntos a la clase que corresponde al suelo. (Axelsson, 2000)

La nube de puntos clasificada representa el producto de datos que contiene la mayor cantidad de información, sin embargo por la cantidad de mediciones que contiene, así como también su naturaleza de espaciamiento irregular, realizar análisis sobre esta es muy complicado. Para facilitar el análisis de datos LiDAR, la información de la nube de puntos se compacta produciendo arreglos regulares (rasters) de datos, similar a una imagen digital en las cuales hay un espaciamiento uniforme entre las filas y columnas, y el valor de cada elemento está relacionado con la información de elevación de los retornos en dicha celda. Un raster de elevación que representa la superficie del suelo sin ningún elemento de vegetación 0 edificaciones se denomina Modelo de Elevación Digital o DEM. Otro tipo de raster que generalmente se produce se denomina Modelo de Superficie Digital o DSM y en este arreglo el valor de cada celda corresponde a la máxima elevación obtenida en cada una. En caso de zonas boscosas, el DSM corresponde a un modelo de la superficie formada por la corona de los árboles.

\section{PRIMERA EXPEDICIÓN}

El cinematógrafo Steve Elkins participó en varias expediciones exploratorias en el Caribe y la Mosquitia Hondureña a mitad de la década de 1990 y conoce de primera mano lo difícil de las condiciones de la jungla y el terreno. En 2011, después de que se hicieron públicos los resultados del mapeo realizado por el Centro Nacional para Mapeo Aéreo (NCALM) de la Fundación Nacional de Ciencia (NSF) de los Estados Unidos en la zona del sitio arqueológico de Caracol en Belice en 2009 (Chase, 2011); Elkins identificó lo valioso que podría ser la tecnología 
de mapeo LiDAR para la exploración arqueológica de la Mosquitia. Elkins contactóo a NCALM y a distintas entidades del gobierno hondureño para realizar un proyecto exploratorio de ciertas zonas de la Mosquitia y al mismo tiempo realizar un documental del mismo. Después de más de un año de investigación, planificación y coordinación se logró realizar la primera expedición para realizar un mapeo exploratorio y realizar el documental al mismo tiempo. La expedición fue dirigida por Steve Elkins con el respaldo legal y económico del también cinematógrafo Bill Benenson y el abogado/activista/tecnólogo/financista Garry Spire. El equipo de exploración incluye al autor y ex -editor del Museo Americano de Historia Natural y Arqueología Douglas Preston, el profesor de media y productor de documentales y programas de televisión Tom Weinberg, al explorador de la Mosquitia Bruce Heinicke y su esposa hondureña Mabel Henríquez. Marvin "Mango" Henríquez fue responsable de la operación de una estación base receptora de GPS en la zona de Dulce Nombre de Culmí, Olancho. También se operaron estaciones GPS en Roatán y una estación GPS autónoma en Trujillo, Colon. El equipo de NCALM basado en la Universidad de Houston estuvo compuesto por el piloto de la aeronave Charles Gross, un piloto de seguridad e ingeniero de sistemas LiDAR Juan Carlos Fernández-Díaz y los ingenieros de procesamiento de datos GPS y LiDAR Michael Sartori y Abhinav Singhania. El equipo de campo de UH/NCALM procesaba los datos en campo y trasmitía imágenes selectas a través del Internet a los profesores William Carter, Ramesh Shrestha y Craig Glenie localizados en Estados Unidos para un análisis complementario más detallado. El equipo de producción del documental estuvo compuesto por Mark Adams, Stephen Graham, Robert Isais. Además de las personas antes mencionadas la expedición no se hubiera podido realizar sin la asistencia de personas como la Antropóloga Alicia Gonzales quien realizó mucho del trabajo de investigación que soportó la expedición; al profesor del Instituto de Tecnología de California (CALTECH) George Rossman asesor científico de Steve Elkins; el arqueólogo Dan Thompson quien realizó análisis de imágenes satelitales de la zona; y Charis Horton encargada de la logística desde Estados Unidos.

Entre el 2 y 12 de Mayo del 2012 se efectuaron un total de 8 vuelos (Roatán - La Mosquitia - La Ceiba - Roatán), con una duración total de 33.6 horas y el láser se operó un total de 8.7 horas. El plan de vuelo y el equipo fue configurado para obtener la mayor penetración a través de la corona de los árboles. Esto incluye una alta frecuencia de pulso laser (PRF) de $133 \mathrm{kHz}$ (133,000 pulsos por segundo), una angosto ángulo de barrido de $\pm 15^{\circ}$ del nadir, divergencia de haz ancha ( 0.8 miliradianes). El plan de vuelo consistía en líneas ortogonales con un traslape lateral 
de $50 \%$ de manera que cada metro cuadrado de jungla se mapea desde 4 ángulos distintos.

Con estas configuraciones, el plan indica una densidad de pulsos laser de casi 26 pulsos $/ \mathrm{m}^{2}$. En total se mapearon 5 áreas $\left(\sim 140 \mathrm{~km}^{2}\right)$ distribuidas dentro de una superficie de $5500 \mathrm{~km}^{2}$ en zonas que se sabe con $100 \%$ de certeza que no han sido científicamente exploradas anteriormente. Se emitieron 3.5 mil millones de pulsos laser, de los cuales se procesaron 2.9 mil millones de pulsos, produciendo un total de 4.5 mil millones de retornos, en promedio 1.54 retornos por pulso. Un $61.1 \%$ de los pulsos produjeron un retorno únicamente, $27.5 \%$ produjeron 2 retornos, $10.3 \%$ produjeron 3 retornos y solo un $2 \%$ produjeron 4 retornos.

\section{HALLAZGOS Y SUS IMPLICACIONES}

En base a las nubes de puntos clasificadas se produjeron modelos de elevación digital (DEM) del suelo (después de remover la vegetación) y modelos de superficie digital (DSM) para representar las copas de los árboles y lo que una cámara óptica registraría como señal desde una vista aérea/espacial. Con los DEMs y DSM se produjeron imágenes de relieve con iluminación sintética (hillshade models), las que muestran el terreno con una exageración vertical y con una iluminación solar proveniente de una altura y azimut determinado. En estas imágenes, que requieren muy leve interpretación humana, es evidente la presencia de montículos, que por su tamaño, ubicación, orientación y distribución entre ellos (muchos en ángulos rectos) se cree que son ruinas arqueológicas. Estas ruinas son evidentes en 2 de las 5 áreas mapeadas, esto no quiere decir que no haya indicios de edificaciones arqueológicas en las otras 3 áreas restantes, con más análisis y más fotointerpretación más estructuras de interés pueden ser detectadas. A diferencia de otros sitios arqueológicos previamente identificados y catálogos en la Mosquitia, el número de estructuras que se encuentran por $\mathrm{km}^{2}$ así como también la extensión superficial que ocupan las estructuras es tal que estos sitios se pudieran catalogar del tamaño de "ciudad" en el contexto de la región mesoamericana.

La Figura 3, muestra las imágenes sintéticas creadas con base en DEM, DSM y la nueve de puntos clasificada de uno de dichos complejos de estructuras. En la parte A) de la figura se muestra una imagen generada con base en DSM, el cual representa los primeros retornos (máxima elevación) detectados por el sistema. Es decir el DSM representa la elevación máxima de la corona de la vegetación. 
La resolución horizontal del DSM es de un metro. En la parte B) de la figura se muestra una imagen generada con base en DEM que se construye con base en los retornos clasificados como suelo y los retornos de vegetación son ignorados, removiendo así la señal de la vegetación. En esta imagen se aprecian unos montículos rectangulares, algunos de los montículos en el lado derecho de la imagen están en ángulos rectos como rodeando un plaza central. En esta imagen se han identificado 9 de estos montículos, sin embargo dentro de la misma imagen hay algunos otros elementos que pueden ser identificados como potenciales modificaciones de terreno por humanos. Para proporcionar una idea del tamaño de estos montículos, se realizó un corte transversal de la nube de puntos en el área que parece ser la plaza rectangular (Ver línea punteada en la Parte B de la figura). Con base en el corte transversal se generó la imagen que se muestra en la parte $\mathrm{C}$ ) de la figura. En esta imagen se puede apreciar la sección transversal de 3 montículos de diferentes tamaños. El montículo mas grande, es de aproximadamente 20 metros de ancho, 5 metros de alto y aproximadamente 60 metros de largo. Entre las dos áreas con estructuras similares se pueden identificar un poco más de 100 elementos peculiares los cuales se deben verificar en campo.

Es importante resaltar que es necesario la verificación in-situ para determinar con $100 \%$ de certeza la naturaleza de estas características superficiales. Actualmente, el arqueólogo Chris Fisher y geógrafo Steve Leisz de Colorado State University están trabajando en conjunto con los investigadores de NCALM/UH en el análisis de los datos LiDAR. Al mismo tiempo Steve Elkins está en el proceso de planificación y coordinación con el gobierno de Honduras para realizar la verificación de campo. La cual es extremadamente complicada por el difícil acceso a las zonas en las que se han encontrado estas estructuras. En caso de que la verificación arroje resultados positivos, sin duda serían hallazgos significativos ya que serían las unidades poblacionales mas grandes conocidas a la fecha en la región de la Mosquitia Hondureña. La posibilidad de utilizar LiDAR de mapeo aéreo para identificar posibles ruinas arqueológicas tiene implicaciones éticas grandes con respecto a las medidas que se tienen que tomar para la protección y preservación del patrimonio cultural y natural de la región. Esta región que ha sido saqueada por depredadores de la flora y fauna y por cazadores de tesoros que saquean reliquias arqueológicas para venderlas al mejor postor en el mercado negro.

Por otro lado, sacando a la luz el carácter excepcional de la Mosquitia, muchas preguntas pueden explorarse más a fondo sobre el poblamiento de esta zona, 
lo cual ha escapado el estudio adecuado. Por ejemplo, algunas de las muchas preguntas que llaman la atención son el grado en que los rasgos culturales más conocidos de América del Norte y América del Sur se pueden identificar, como la delimitación o ampliación de lo que se designa como Mesoamérica con sus juegos de pelota y plazas; específicos rasgos compartidos con otras culturas del Caribe; 0 las influencias de caminos pavimentados de las culturas muy tempranas de Costa Rica y Panamá que comparten una raíz lingüística macro-Chibcha con los Pech de la Mosquita, o los restos de cultivos como el maíz, la yuca o el cacao, o más importante, la identificación de un complejo único y diferente y la organización social de las culturas de la región. Hoy en día esto son hipótesis y conjeturas, basada en lo poco que se conoce de los alrededores y el trabajo de los pocos que han asumido el reto de la remota región. Pero más pruebas concretas, más estudios y la participación de equipos de especialistas de varias disciplinas contribuirán en gran medida a identificar algunas nuevas piezas que faltan para darnos una imagen más amplia del mapa político y cultural de la Mosquitia.

\section{BIBLIOGRAFÍA}

- Axelsson, P. (2000), "DEM generation from laser scanner data using adaptive tin models."En International Archives of Photogrammetry and Remote Sensing, XXXIII, Part B3, 85-92

- Begley, C. (2002), "El Clásico Tardío y el Postclásico Temprano en el Oriente de Honduras." En XV Simposio de Investigaciones Arqueológicas en Guatemala 2001 (editado por J.P. Laporte, H. Escobedo y B. Arroyo), pp.36-47.

- Begley, C. (2007), "Intercambio Interregional, Conexiones Externas, y Estrategias de Poder en el Oriente de Honduras durante Periodos V y VI." en Arqueología del Área Intermedia, Bogotá, Colombia.Chase, A. et al. (2011): "Airborne LiDAR, archaeology, and the ancient Maya landscape at Caracol, Belize" en Journal of Archaeological Science, Vol. 38, Is. 2, February 2011, pg 387-398.

- Fernandez-Diaz, J.C. et al. (2013): Chapter 31: "LiDAR Remote Sensing", en Handbook of Satellite Applications; Pelton, Madry y Camacho Lara (Edt). New York, Springer. 
- Guenther, G.; Goodman, L.; Enabnit, D.; Swift, R.; Thomas, R. (1978), "Laser Bathymetry for Near Shore Charting Application (Preliminary Field Test Results)", en OCEANS '78 , vol., no., pp.390-396, doi: 10.1109/OCEANS.1978.1151121

- Hickman, G.D. y Hogg, J.E. (1969): "Application of an Airborne Pulsed Laser for Near Shore Bathymetric Measurements", en Remote Sensing of the Environment, Vol 1, No 1, P 47-58.

- Lara-Pinto, G. (2006): "La Investigación Arqueológica En Honduras: Lecciones Aprendidas para una Futura Proyección." en Revista Pueblos y Fronteras digital, No 2. UNAM, México

- Mugnier, C.J. (1999): "The Republic of Honduras" en Grids \& Datums, Photogrammetric Engineering \& Remote Sensing, P 754-755. July 1999.

- Smithsonian Institution (SF): "Selections from the Field Journal of William Duncan Strong (Honduras, 1933)" http://www.nmnh.si.edu/naa/features/strong.htm

- Yakam-Simen, F. Nezry. E y Ewing J. (1999): "A legendary lost city found in the Honduran tropical forest using ERS-2 and JERS-1 SAR imagery". En Proceedings of the Geoscience and Remote Sensing Symposium, 1999. IGARSS ' 99 5: 2578 - 2580 vol. 5 . 
A)

B)

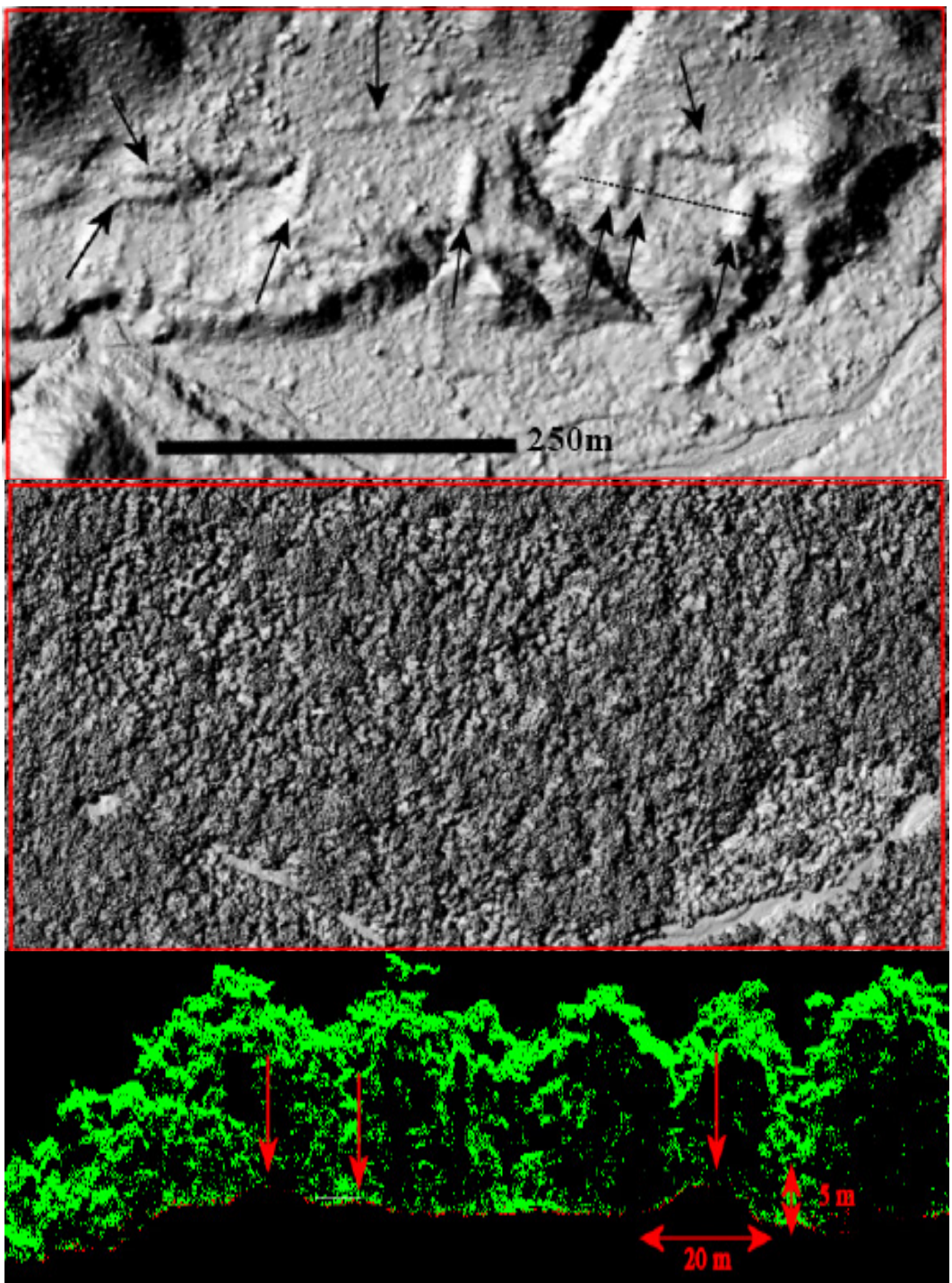

Figura 3. Imágenes sintéticas basadas en: A) el DSM (corona de la vegetación), B) DEM (suelo) y C) Perfil extraído de la nueve de puntos clasificada (verde = vegetación, rojo = suelo) 